\title{
Speckle-based at-wavelength metrology of x-ray optics at Diamond Light Source
}

Hongchang Wang, Tunhe Zhou, Yogesh Kashyap, Kawal Sawhney

Hongchang Wang, Tunhe Zhou, Yogesh Kashyap, Kawal Sawhney, "Specklebased at-wavelength metrology of $\mathrm{x}$-ray optics at Diamond Light Source,"

Proc. SPIE 10388, Advances in Computational Methods for X-Ray Optics IV, 103880I (23 August 2017); doi: 10.1117/12.2274781

SPIE Event: SPIE Optical Engineering + Applications, 2017, San Diego, California, United States 
Invited Paper

\title{
Speckle-based at-wavelength metrology of X-ray optics at Diamond Light Source
}

\author{
Hongchang Wang*, Tunhe Zhou, Yogesh Kashyap† and Kawal Sawhney \\ Diamond Light Source Ltd, Harwell Science and Innovation Campus, Didcot, OX11 0DE, UK
}

\begin{abstract}
To achieve high resolution and sensitivity on the nanometer scale, further development of X-ray optics is required. Although ex-situ metrology provides valuable information about X-ray optics, the ultimate performance of X-ray optics is critically dependent on the exact nature of the working conditions. Therefore, it is equally important to perform in-situ metrology at the optics' operating wavelength ('at-wavelength' metrology) to optimize the performance of X-ray optics and correct and minimize the collective distortions of the upstream beamline optics, e.g. monochromator, windows, etc. Speckle-based technique has been implemented and further improved at Diamond Light Source. We have demonstrated that the angular sensitivity for measuring the slope error of an optical surface can reach an accuracy of two nanoradians. The recent development of the speckle-based at-wavelength metrology techniques will be presented. Representative examples of the applications of the speckle-based technique will also be given - including optimization of X-ray mirrors and characterization of compound refraction lenses. Such a high-precision metrology technique will be extremely beneficial for the manufacture and in-situ alignment/optimization of X-ray mirrors for next-generation synchrotron beamlines.
\end{abstract}

Keywords: Metrology, at-wavelength, in-situ characterization, near- field speckle, synchrotron radiation

\section{INTRODUCTION}

Over the last few decades, significant progress has been made in improving both the optical quality and metrology accuracy of X-ray optics. Nowadays, the state of art of the deterministic surface finishing technique, such as, Elastic Emission Machining (EEM) [1], has pushed the mirror slope error below 50 nrad. Nevertheless, further improvements in the precision of manufacturing modern optical components are often limited by the accuracy of available metrology techniques. Therefore, it in turn demands advanced metrology techniques to be developed to further push the performance and manufacturing limits of advanced optics. Modern deflectometry instruments such as the Nanometer Optical Measuring system (NOM) demonstrate high accuracy and have become indispensable tools for any metrology labs at synchrotron facilities [2,3]. The metrological accuracy of mirror slope error measurements has been be pushed into tens of nrad [4]. In addition, the Hartmann wavefront sensor has been implemented into the ex-situ metrology, and two-dimensional mirror slope errors can be measured [5]. However, the ultimate performance of the optics is often affected by factors such as X-ray beam alignment, mechanical and thermal drifts, and vibrations. Therefore, the in-situ and at-wavelength metrology methods are considered the best pathway to overcome this limitation and surpass the present optics performance [6-9]. As well as optical tests in the metrology labs, it is becoming increasingly important to optimize and characterize X-ray optics under actual beamline operating conditions.

Recently, many in-situ metrology methods have been developed for aligning or optimizing the X-ray mirrors [9-12]. Nevertheless, they inherently suffer from low spatial or angular sensitivity, slow optimization process or complicated setup. In contrast, the recently developed X-ray speckle-based at-wavelength metrology technique has shown great potential due to its simple experimental setup, high angular accuracy and moderate requirement of mechanical stability and transverse coherence [8, 13-18]. These techniques have been established and further developed at Diamond Light Source (DLS) beamline B16 and is increasingly used to characterize X-ray optics.

*Hongchang.wang@diamond.ac.uk; phone +44 123 577-8754

$†$ Present address: Technical Physics Division, Bhabha Atomic Research Centre, Mumbai-400085, India 
Importantly, an unprecedented angular sensitivity in the range of sub-ten nrad for measuring the slope error of an optical surface has been demonstrated $[14,15]$. Such a super precision metrology technique will be beneficial to the manufacturers of polished mirrors and also in optimization of beam shaping during experiments. Here, we present an overview for the recent development of X-ray speckle-based techniques at DLS and show the applications of this atwavelength metrology method for characterizing compound refractive lenses (CRLs), optimizing X-ray mirrors, and measuring beam coherence length.

\section{X-RAY SPECKLE-BASED TECHNIQUE AND EXPERIMENTAL SETUP}

\subsection{Introduction of $\mathrm{X}$-ray speckle-based technique}

The principle of speckle-based technique is to track the displacement of near-field speckles, which arise from the mutual interference of light generated by imperfections and the rough structure of the objects located in the coherent light path. In the near-field regime, the speckles can be generated by putting a diffuser (such as abrasive paper or filter membrane) into the X-ray beam. The average particle size of the diffuser is usually a few microns, and the generated speckles can be resolved using a high-resolution X-ray detector. Based on the sample under test, the technique can be simply classified into two modes: differential mode and self-correlation mode $[14,16]$.

For weakly focusing optics, such as single CRL, the footprint of X-ray beam -in presence and absence of optics remains same. In the differential mode, two sets of images with and without the sample in the beam are collected. Here, each set of image can be single image or a stack of images based on the requirement on angular sensitivity. If only single speckle image is used, a surrounding area for each pixel is used for tracking the speckle displacement with a digital image correlation algorithm capable of subpixel accuracy [19]. Even though this method is fast, the spatial resolution and angular sensitivity is compromised. To overcome this issue, both two dimensional (2D) scan and one dimensional (1D) scan have been developed [18, 20]. Recently, the speckle vector tracking and unified modulated pattern analysis approaches have been proposed to reduce the number of speckle images [21, 22].

In contrast, for strongly focusing optics, the footprint of X-ray beam is significantly changed after the optics inserted into the beam. Therefore, it is difficult to perform cross-correlation between the direct reference beam and the "zoomed" beam, after inserting strong focusing optics. In this case, only a single stack of images are collected by scanning the diffuser with the optics in the beam. Once the single stack of images are acquired, the speckle patterns can be built from all the speckle images for the two nearby rows of pixels [8]. The speckle displacement can be retrieved from the two generated speckle patterns. Instead of retrieving the first derivative of wavefront phase from the differential mode, the secondary derivative of wavefront phase is derived from the self-correlation mode [14]. The wavefront slope is then calculated by $1 \mathrm{D}$ or $2 \mathrm{D}$ integrations from the derived secondary derivative of wavefront phase [16]. It should be noted that the measured wavefront slope error from the self-correlation mode includes both the defect from the optics under test and the upstream incoming wavefront error. If the incoming wavefront error is much less than the defects from the optics under test, the measured wavefront slope error can be used to quantify the defect of the optics. The ultimate accuracy for the absolute slope error is limited by the imperfection of incoming wavefront. Nevertheless, the measured wavefront error can still be used to evaluate quality of the X-ray beam since the optics is tested under actual beamline operating conditions.

\subsection{Experimental setup}

The experiment for the development of speckle-based technique was carried at the Diamond Light Source Test beamline B16 [23]. The monochromatic X-ray can be selected by either a Si (111) double-crystal monochromator (DCM) or a double multilayer monochromator (DMM). As shown in Fig. 1, an X-ray mirror under test was mounted on a motorized stage, located at $47 \mathrm{~m}$ from the source. A diffuser was mounted on a piezo stage and was placed downstream from the mirror. The scanning step size for the diffuser was normally sub-micron. A set of images was collected with a 2D detector, and the acquisition time is about a few seconds for each image depending on the experimental setup. The sensitivity for measuring the local wavefront radius of curvature can be increased by putting the diffuser closer to the focal plane. In order to resolve the speckle features and increase angular sensitivity, the detector was located at a large distance (such as $1000 \mathrm{~mm}$ ) downstream from the focusing optics. 


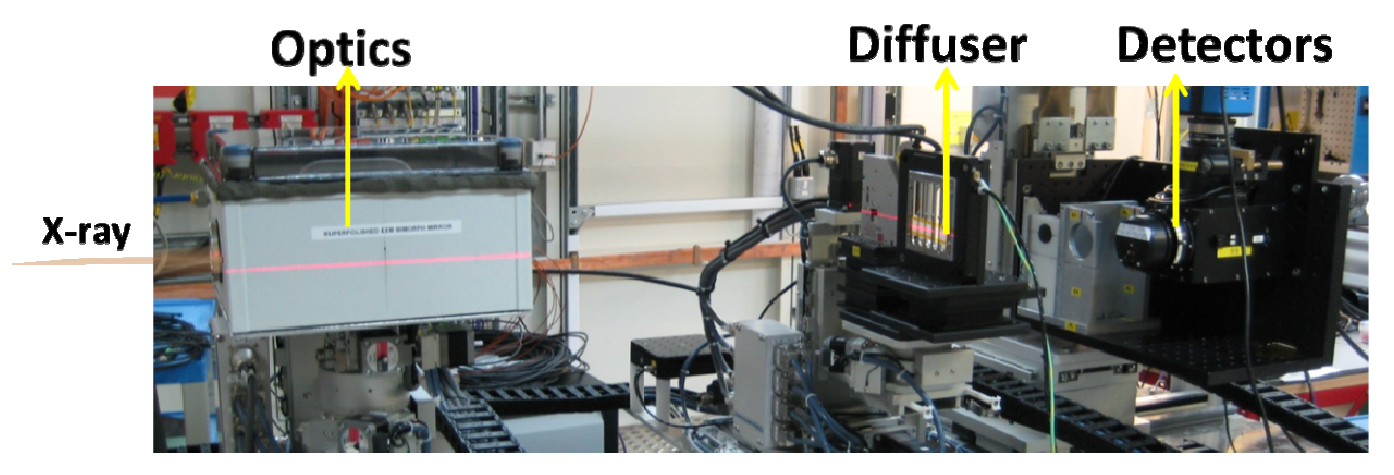

Figure 1. Photograph of a typical experimental setup installed on B16 beamline. Here, an X-ray mirror is installed upstream of the diffuser stage, and CCD detector is mounted downstream of the diffuser stage.

\section{APPLICATIONS FOR AT-WAVELENGTH METROLOGY}

\subsection{Characterization of a compound refractive lens}

As a demonstration of the capabilities of the speckle-based technique for in-situ metrology, a 2D CRL was characterized using the differential mode approach [19]. The CRL was made of beryllium and had a parabolic shape with a design radius of curvature $200 \mu \mathrm{m}$ at the apex [24]. The beam energy was set to $15 \mathrm{keV}$ using a $\mathrm{Si}(111)$ from DCM. As illustrated in Figure 2, applying the differential mode, two sets of images were recorded: (a) one without the CRL in the X-ray beam and (b) the other with the lens. For single image mode, a cross-correlation algorithm with sub-pixel accuracy was employed to calculate the speckle displacement between the two surrounding subsets for each pixel [25]. By dividing the displacement by the distance between the lens and the detector, both the horizontal and the vertical wavefront gradient can be calculated. As shown in Figure 2(c), the derived vertical wavefront gradient image from single image mode seems noisy-. For a 2D raster scan, $33 \times 33$ images were acquired while scanning the diffuser with $1 \mu \mathrm{m}$ step size [18]. Figure 2(d) shows the derived vertical wavefront gradient, and significant improvement on spatial and angular resolutions have been achieved compared the one from single image mode. For speckle-based technique, three predominant parameters, namely, the tracking accuracy, the diffuser to detector distance and the scanning step size, determine the angular sensitivity of the speckle scanning technique. In addition, the angular sensitivity can be further improved by averaging several series of speckle images to reduce random noise. However, it will be -time-consuming to achieve high spatial and angular resolution by performing 2D raster scan. To overcome this issue, 1D scan mode was proposed [15]. In this case, the diffuser is only required to scan along one direction. As shown in Figure 2(e), the spatial resolution is as good as the one from 2D scan, while only 40 images are collected. It should be noted that the angular sensitivity is proportional to the step size along scan direction, while the one along the orthogonal direction is related to the detector pixel size. The corresponding aberrations in the parabolic form can be retrieved by subtracting the best-fit linear term from the wavefront gradient [15]. Such precise measurement of the refractive properties is essential for further improvement to the fabrication process, to achieve smaller X-ray focal spots. 


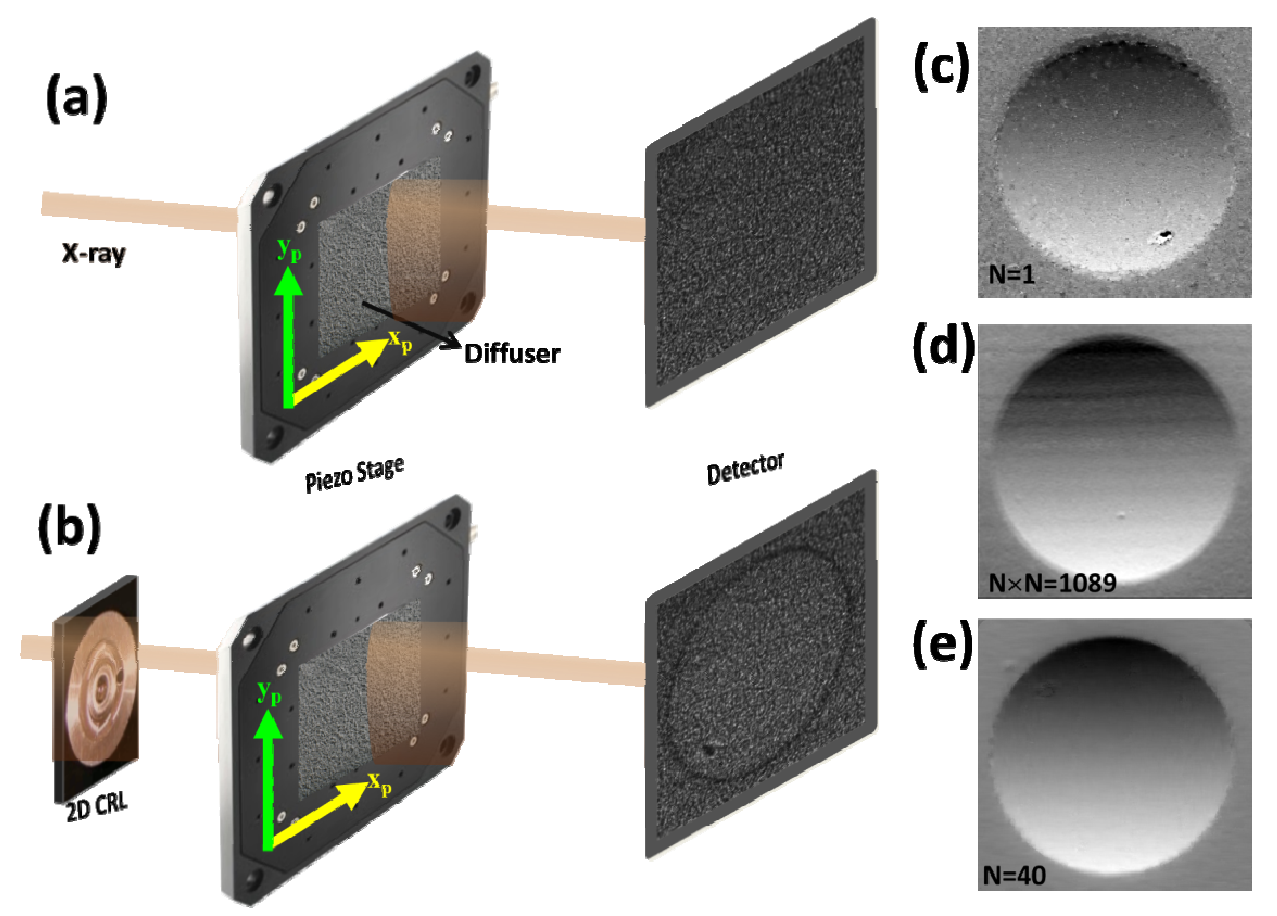

Figure 2. Schematic of the experimental setup for speckle-based technique with the differential mode. (a) Reference speckle pattern is collected without sample in the beam. (b) The test sample (a 2D CRL) is placed upstream of the diffuser, which is mounted on a precision piezo translation stage. The speckle pattern is recorded using a high resolution X-ray area detector. Derived vertical wavefront gradient of the 2D CRL from (c) single image mode, (d) two dimensional scan mode and (e) one dimensional scan mode

\subsection{Optimization of a bimorph X-ray mirror}

Active optics, such as bimorph and mechanically bendable mirrors, are widely used for beam shaping since they permit a wide choice of focal lengths and beam sizes. Here, we demonstrate that the speckle-based technique can be used for quick optimization of a bimorph mirror [14]. The bimorph mirror was made from a silica substrate with a preformed elliptical surface and had 8 piezo electrodes cemented underneath to allow the curvature to be varied in order to correct the figure error [26]. The surface of the bimorph mirror was pre-polished to an ellipse with a focal length of $f=0.4 \mathrm{~m}$ with grazing angle of incidence at the center of the mirror of $\theta=3 \mathrm{mrad}$. As illustrated in Figure 3, the mirror's slope error introduces errors to the wavefront, thus broadening the beam size at the focal point F. The bimorph mirror's slope error and hence the wavefront error can be minimized by applying appropriate voltages to the mirror's piezo actuators. The diffuser's position transverse to the beam was scanned with a step size $\mu$ using a piezo stage. Once the diffuser scan was completed, a speckle pattern was built up from the $i^{\text {th }}$ rows of all the speckle images in order of diffuser's positions. The second speckle pattern was generated the same way from the neighboring row of all the speckle images. With the self-correlation mode, the cross-correlation was then applied to the two generated speckle patterns, and the calculated speckle delay could be used for deriving the second derivative of the wavefront, namely the inverse of its local radius of curvature $R$. To determine the optimal voltages which minimize slope errors, a stack of speckle images were first collected with all piezo voltages set to $0 \mathrm{~V}$. One stack of speckle images were then collected in the same way for each subsequent scan by increasing the voltage on the $j^{\text {th }}$ electrode by $400 \mathrm{~V}$. The piezo response function (PRF) was then derived by subtracting $1 / \mathrm{R}$ extracted from the $j^{\text {th }}$ to $(j-1)^{\text {th }}$ measurement. Here, the PRF was determined in terms of $1 / R$ for fast optimization and convergence compared to the one determined from wavefront slope. After deriving the PRF, the bimorph mirror can be optimized to achieve the best focus with derived voltage. As demonstrated in Figure 
3(c), the bimorph mirror can also provide different beam sizes at the original focal plane $\mathrm{F}$ by changing its surface to a different ellipse. In this case, the focal length $q$ will be changed. If the distance $L$ between the mirror and the detector is fixed, the measured wavefront radius $R$ at the detector plane will be changed with $q$.

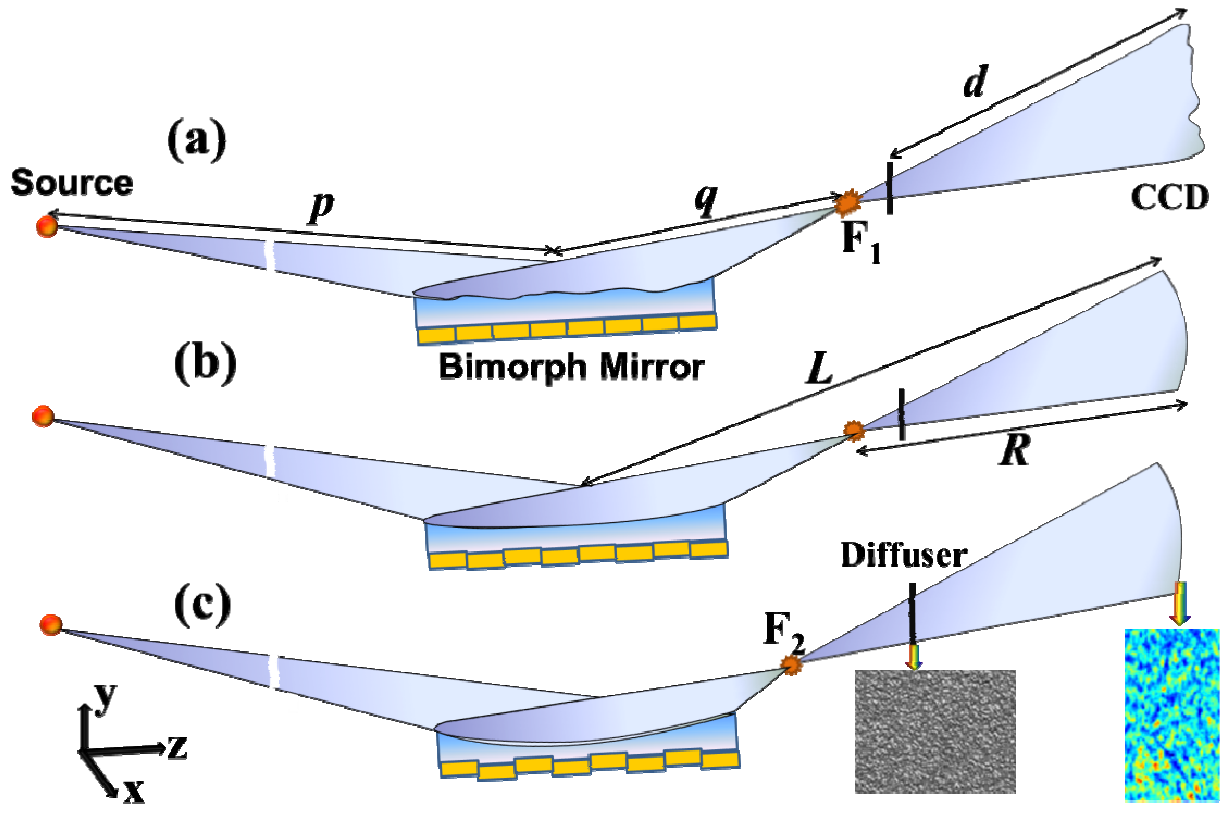

Figure 3. Optical layout of the in-situ optimization of a bimorph mirror for three cases (a) bimorph voltages at $0 \mathrm{~V}$, (b) bimorph voltages for focused operation after the $1^{\text {st }}$ iteration, (c) first optimization for bimorph defocused.

To verify the focus and defocus optimization, a series of images were taken by the high-resolution camera PCO 4000 CCD camera along the beam direction with $1 \mathrm{~mm}$ step size. Figure 4 shows the intensity profiles around the focal plane of the bimorph mirror for the three cases. There are strong oscillations on the edge of the beam profile for case (a) that are mainly due to the large mirror slope error before optimization. The beam intensity profile became much more uniform for case (b) at the focal plane after the optimization. Figure 4(c) show the focal position moving upstream towards the mirror while the intensity profile remains uniform at the original focal plane. The generation of a "tophat" beam is highly desired by many X-ray synchrotron users because it can significantly reduce the radiation damage. It should be highlighted that the diffuser can be placed downstream of test optics without accessing the focal position. Based on this advantage, we have developed a speckle-based portable device for in-situ (at-wavelength) metrology of Xray optics at DLS [13]. This fast, compact and accurate speckle-based device can be easily adopted on a variety of operational beamlines. 


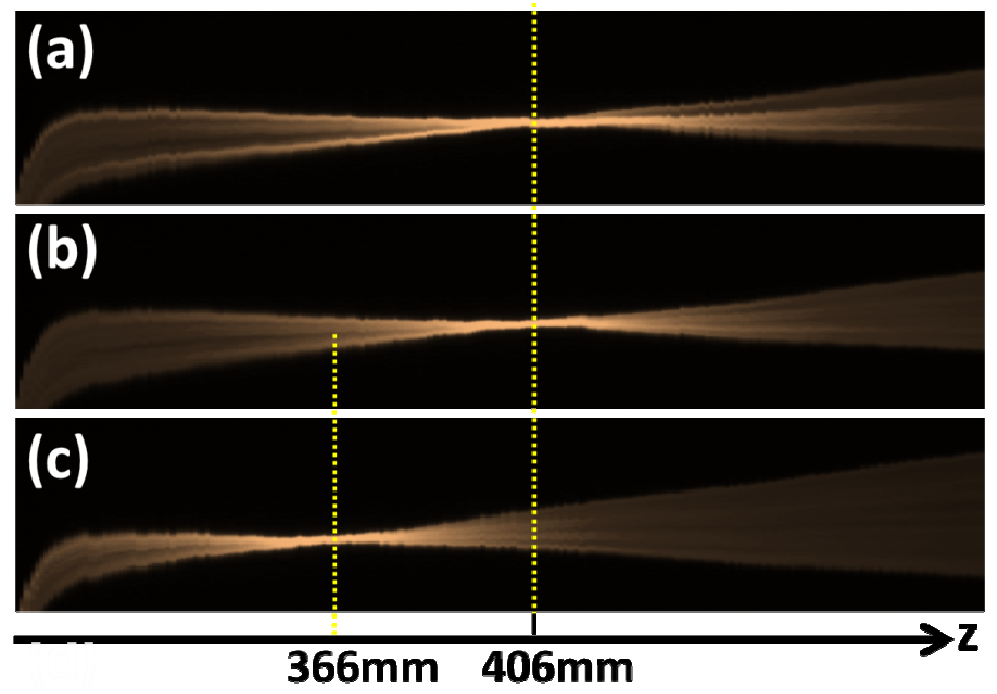

Figure 4. The intensity profiles as a function of distance $\mathrm{z}$ in $\mathrm{mm}$ from the bimorph mirror for the three cases, (a) bimorph voltages at $0 \mathrm{~V}$, (b) bimorph voltages for focused operation after $1^{\text {st }}$ iteration, (c) first optimization for bimorph defocused. The yellow dotted lines mark the focal planes.

\subsection{Metrology of an X-ray mirror}

The speckle-based technique has also been used as a deflectometer for the measurement of 2D slope error profiles of Xray mirrors [16] using the self-correlation mode. For the above example, the speckle pattern was generated from a stack of speckle images for a row of pixels, and only 1D mirror slope error can be derived by averaging the mirror slope error along mirror width. In fact, the speckle pattern is inherently two-dimensional, and is expected to move in both horizontal and vertical directions for the spherical incidence wavefront even if the diffuser is translated along single direction. Therefore, instead of performing two orthogonal scans, the horizontal displacement can be retrieved from the same dataset for the vertical scan, and vice versa. Although the 2D mirror slope error can be calculated by simply performing 1D integration, artefacts will be produced along the direction of the integration due to propagation of statistical errors [27]. In this study, the diffuser was mounted upstream of the mirror, which is the same bimorph mirror used in above example.

As shown in Figure 5, the 1D slope error curve (a) and integrated 2D slope error map (b) are compared with all the piezo voltages set to $0 \mathrm{~V}$ to the bimorph mirror. 1D slope error curve shows the similar trend as the variation profile from the 2D slope error map, and the root mean square of slope errors for 1D and 2D slope error are both around $1.7 \mu \mathrm{rad}$. The 1D slope error curve can only provide the averaged slope error information for the mirror, while the 2D slope error map can reveal the slope error variation along the sagittal direction (mirror width). Moreover, the spatial resolution of the measured slope for sagittal and tangential error is $0.12 \mathrm{~mm}$ and $0.27 \mathrm{~mm}$, respectively. They are both in the midfrequency region. Such information is expected to be extremely valuable for studying the contribution of the small- and large-angle scattering of X-rays from the mirror surface [28]. It may be mentioned that it is very challenging for current ex-situ metrology techniques to measure slope error in the mid-frequency region. In addition, the high angular sensitivity can also be achieved for the 2D slope error measurement. The measurement of 2D slope error profiles of X-ray mirrors can provide valuable information on optical aberrations of the X-ray aspherical mirrors, such as ellipsoidal and toroid mirrors, which are difficult to obtain using conventional ex-situ visible light metrology techniques. 


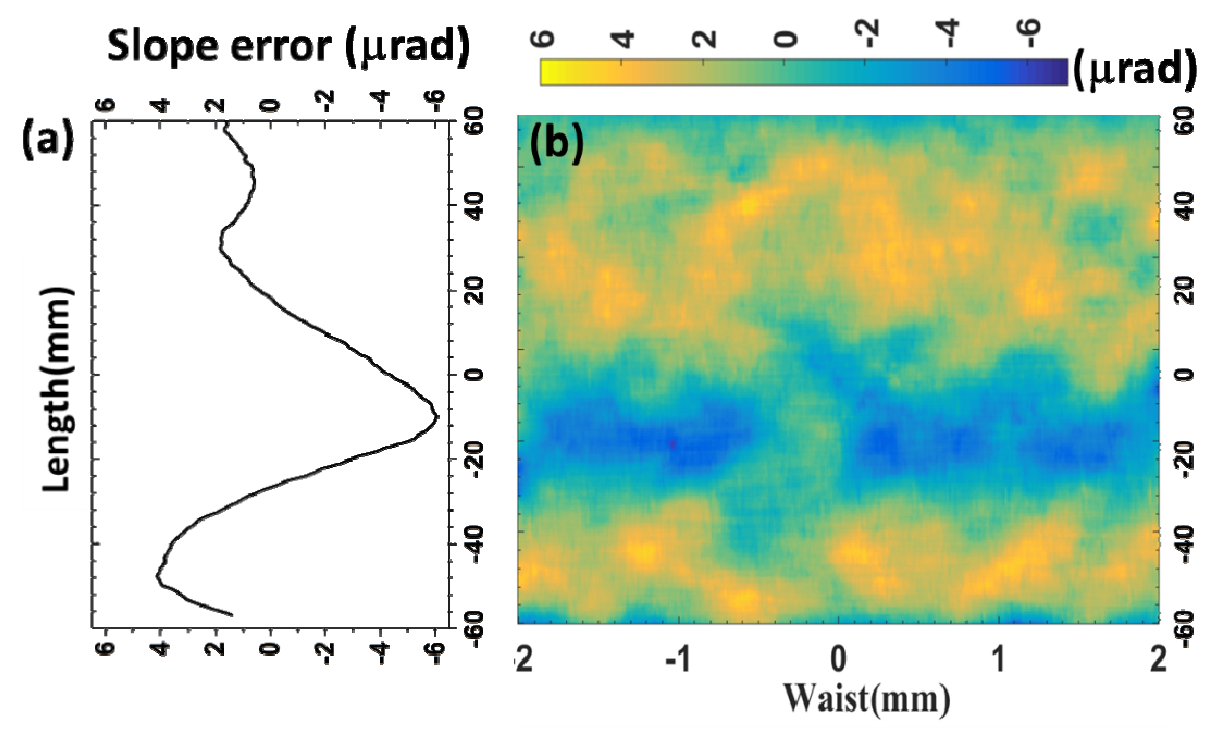

Figure 5. (a) Measured 1D and (b) 2D mirror slope error from the speckle-based technique.

\subsection{Measurement of coherence length}

The measurement of the transverse coherence of X-ray beams is extremely important not only for understanding the source properties, but also to study the impact of X-ray optics. The transverse coherence properties can be characterized by using near-field speckle approach [17], which does not require moving the detector at multiple distances. In the near-field regime, the power spectrum of the normalized scattered signal can be decomposed as the product of the near-field transfer function and the power spectrum of the absolute scattering intensity of the diffuser $[17,18]$. The transfer function includes the distance-dependent Talbot effect, partial coherence effect due to the incident wavefront and the detector response function. The detector response function is determined experimentally, and it can be used as an input parameter during the analysis. The two-dimensional transverse coherent lengths of the source can then be derived from the fitting of the speckle power spectrum.

The experiments were performed at the DLS B16 Test beamline. Images of the speckle pattern were collected using a high-resolution $\mathrm{X}$-ray microscope placed downstream of the diffuser. The double multilayer monochromator with selected energy of $15 \mathrm{keV}$ was used. The measurements were carried out by changing the vertical electron-beam coupling in the $0.16 \%-1.33 \%$ range to facilitate the experiment at different vertical source sizes. As shown in Figure 6 (a), a raw speckle image collected at $0.3 \%$ electron beam coupling conditions, and the horizontal stripes are due to the multilayer monochromator used for collecting the images. In principle, only a single image needs to be acquired for this technique. However, in order to improve signal-to-noise ratio and remove contributions of stray signal and detector noise, the data was collected by raster scanning the diffuser stage and generating a multiple dataset. The data was cropped to small region of interest and the correction for the background data is achieved by averaging of all the independent scans. Once the raw data was generated and background was properly removed, as shown in Figure 6 (b), the 2D power spectrum of the near-field speckle was obtained via Fourier transform. As expected, the number of rings visible in vertical direction is more than double compared to in the horizontal since the vertical transverse coherence length was larger than the horizontal one, and it agrees well with the property of bending magnetic source for beamline B16. The measured coherence length is $11 \mu \mathrm{m}(\mathrm{H})$ and $31 \mu \mathrm{m}(\mathrm{V})$, slightly less than the theoretical values, and it indicates that the imperfection of the multilayer moderately deteriorate the beamline's coherence. The corresponding 2D power spectrum at 1.3\% electron beam coupling conditions is shown in Figure 6 (c). The fringes observed in the power spectrum in low coupling mode $(0.3 \%)$ extend to much higher frequencies in the vertical direction than in high coupling mode $(1.3 \%)$, thereby verifying that only vertical source size is affected. As expected, the vertical coherence length increases when the vertical coupling is decreased, while the horizontal coherence length remains nearly the same. It should be emphasize that small pixel size and detector point spread function as well as long propagation distance will be beneficial for retrieving high coherence length from undulator beamlines. 

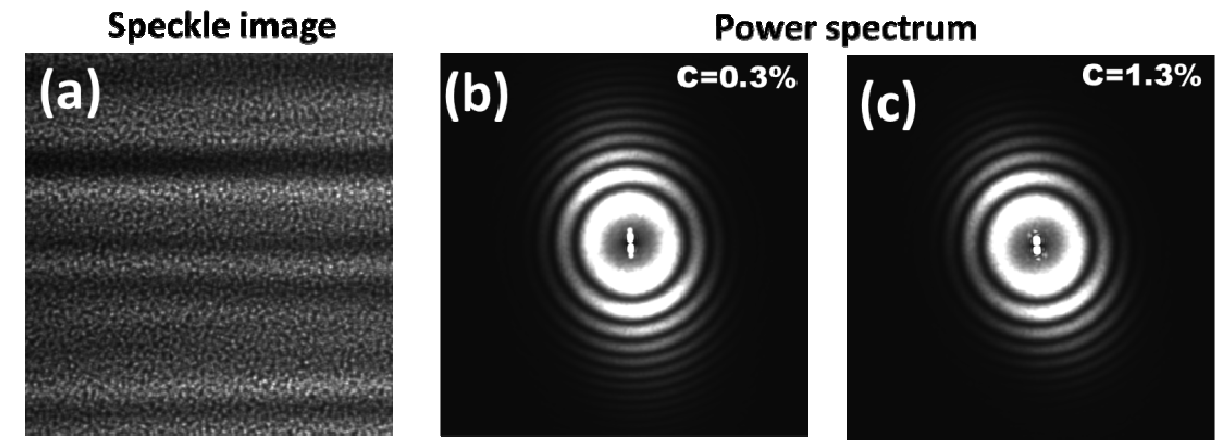

Figure 6. (a) Raw speckle image. The corresponding averaged power spectrum with an e-beam coupling (C) of $0.3 \%$ (b)and $1.3 \%$ (c).

\section{SUMMARY}

The X-ray speckle-based technique is established and further developed at the Diamond Light Source beamline B16. The technique is compact, robust and has no stringent requirements on longitudinal and transverse coherence. An unprecedented angular sensitivity in the range of sub-ten nrad for measuring the slope error has been demonstrated, and the spatial resolution can be pushed to the mid-frequency region. Furthermore, we have shown that either fast or high resolution can be achieved by using single image or multiple images mode based on the requirement for test optics. Importantly, the X-ray speckle-based technique fulfills the requirement for optimization of future diffraction-limited optics, and it is valuable for the improvement of the manufacture and in-situ optimization of the new generation of X-ray optics. It thereby complements other phase retrieval methods for in-situ and at-wavelength metrology, and has the potential for the routine use of future nano-focusing optics. The advanced in-situ metrology method could open new possibilities for characterization and optimization of X-ray optics for next-generation synchrotron radiation sources and astronomical telescopes.

\section{Acknowledgments}

This work was carried out with the support of the Diamond Light Source Ltd UK. We would like to acknowledge Andrew Malandain and Ian Pape for their technical assistance.

\section{REFERENCES}

[1] K. Yamauchi, H. Mimura, K. Inagaki et al., "Figuring with subnanometer-level accuracy by numerically controlled elastic emission machining," Review of Scientific Instruments 73(11), 4028-4033 (2002).

[2] F. Siewert, T. Noll, T. Schlegel et al., "The Nanometer Optical Component Measuring Machine: a new Sub-nm Topography Measuring Device for X-ray Optics at BESSY," AIP Conference Proceedings, 705(1), 847-850 (2004).

[3] S. G. Alcock, K. J. S. Sawhney, S. Scott et al., "The Diamond-NOM: A non-contact profiler capable of characterizing optical figure error with sub-nanometre repeatability," Nuclear Instruments and Methods in Physics Research Section A: Accelerators, Spectrometers, Detectors and Associated Equipment, 616(2-3), 224-228 (2010).

[4] F. Siewert, J. Buchheim, S. Boutet et al., "Ultra-precise characterization of LCLS hard X-ray focusing mirrors by high resolution slope measuring deflectometry," Optics Express 20(4), 4525-4536 (2012).

[5] M. Idir, K. Kaznatcheev, G. Dovillaire et al., "A 2 D high accuracy slope measuring system based on a Stitching Shack Hartmann Optical Head,” Optics Express 22(3), 2770-2781 (2014).

[6] H. Wang, K. Sawhney, S. Berujon et al., "X-ray wavefront characterization using a rotating shearing interferometer technique," Opt. Express, 19(17), 16550-16559 (2011).

[7] K. Sawhney, H. Wang, J. Sutter et al., "At-wavelength Metrology of X-ray Optics at Diamond Light Source," Synchrotron Radiation News, 26(5), 17-22 (2013).

[8] S. Berujon, H. Wang, S. Alcock et al., "At-wavelength metrology of hard X-ray mirror using near field speckle," Optics Express 22(6), 6438-6446 (2014). 
[9] J. Sutter, S. Alcock, and K. Sawhney, "In situ beamline analysis and correction of active optics," Journal of Synchrotron Radiation, 19(6), 960-968 (2012).

[10] M. Idir, P. Mercere, M. H. Modi et al., "X-ray active mirror coupled with a Hartmann wavefront sensor," Nuclear Instruments and Methods in Physics Research Section A: Accelerators, Spectrometers, Detectors and Associated Equipment, 616(2-3), 162-171 (2010).

[11] H. Yumoto, H. Mimura, S. Matsuyama et al., "At-wavelength figure metrology of hard x-ray focusing mirrors," Review of Scientific Instruments, 77(6), 063712-6 (2006).

[12] H. Wang, K. Sawhney, S. Berujon et al., "Fast optimization of a bimorph mirror using x-ray grating interferometry," Optics Letters 39(8), 2518-2521 (2014).

[13] Y. Kashyap, H. Wang, and K. Sawhney, "Development of a speckle-based portable device for in situ metrology of synchrotron X-ray mirrors," Journal of Synchrotron Radiation, 23(5), 1131-1136 (2016).

[14] H. Wang, J. Sutter, and K. Sawhney, "Advanced in situ metrology for x-ray beam shaping with super precision," Optics Express 23(2), 1605-1614 (2015).

[15] H. Wang, Y. Kashyap, and K. Sawhney, "Speckle based X-ray wavefront sensing with nanoradian angular sensitivity," Optics Express 23(18), 23310-23317 (2015).

[16] H. Wang, Y. Kashyap, D. Laundy et al., "Two-dimensional in situ metrology of X-ray mirrors using the speckle scanning technique,” Journal of Synchrotron Radiation, 22(4), 925-929 (2015).

[17] Y. Kashyap, H. Wang, and K. Sawhney, "Two-dimensional transverse coherence measurement of hard-x-ray beams using near-field speckle," Physical Review A, 92(3), 033842 (2015).

[18] S. Berujon, H. Wang, and K. Sawhney, "X-ray multimodal imaging using a random-phase object," Physical Review A, 86(6), 063813 (2012).

[19] S. Berujon, E. Ziegler, R. Cerbino et al., "Two-Dimensional X-Ray Beam Phase Sensing," Physical Review Letters, 108(15), 158102 (2012).

[20] H. Wang, Y. Kashyap, and K. Sawhney, "From synchrotron radiation to lab source: advanced speckle-based Xray imaging using abrasive paper," Scientific Reports, 6, 20476 (2016).

[21] M.-C. Zdora, P. Thibault, T. Zhou et al., "X-ray Phase-Contrast Imaging and Metrology through Unified Modulated Pattern Analysis," Physical Review Letters 118(20), 203903 (2017).

[22] S. Berujon, and E. Ziegler, "X-ray Multimodal Tomography Using Speckle-Vector Tracking," Physical Review Applied, 5(4), 044014 (2016).

[23] K. J. S. Sawhney, I. P. Dolbnya, M. K. Tiwari et al., "A Test Beamline on Diamond Light Source," AIP Conference Proceedings, 1234(1), 387-390 (2010).

[24] B. Lengeler, C. Schroer, J. Tummler et al., "Imaging by parabolic refractive lenses in the hard X-ray range," Journal of Synchrotron Radiation, 6(6), 1153-1167 (1999).

[25] B. Pan, H.-m. Xie, B.-q. Xu et al., "Performance of sub-pixel registration algorithms in digital image correlation," Measurement Science and Technology, 17(6), 1615 (2006).

[26] K. J. S. Sawhney, S. G. Alcock, and R. Signorato, "A novel adaptive bimorph focusing mirror and wavefront corrector with sub-nanometre dynamical figure control." 7803, 780303-780303-9.

[27] C. Kottler, C. David, F. Pfeiffer et al., "A two-directional approach for gratingbased differential phase contrastimaging using hard x-rays," Opt. Express, 15(3), 1175-1181 (2007).

[28] J. E. Harvey, K. L. Lewotsky, and A. Kotha, "Effects of surface scatter on the optical performance of x-ray synchrotron beam-line mirrors," APPLIED OPTICS, 34(16), 3024-3032 (1995). 